\title{
Legado arqueológico, academicismo e historia en el setecientos español
}

\author{
Maria Teresa Nava Rodríguez
}

\section{REFLEXIONES INTRODUCTORIAS}

En una obra muy reciente, editada hace apenas un año, el historiador francés Barret-Kriegel ', recordando una frase de Momigliano, llama la atención sobre un hecho que invita a la reflexión: "Je voudrais pouvoir me contenter - nos dice este segundo autor- de renvoyer la lecteur à une "histoire des études antiquaires" mais il n'en existe pas..." ${ }^{2}$. Esta afirmación, aún no siendo interpretada en términos absolutos, resulta no obstante ilustrativa a la hora de constatar cómo la separación entre los estudios "de anticuario" y las investigaciones históricas se va haciendo cada vez más acusada a medida que transcurren los siglos $x \mid x$ y $X X$, incidiendo muy negativamente en la atención prestada por los historiadores a temas relacionados con la historiografía del siglo XVIII.

Pero no sólo la historia de la historiografía ha resultado perjudicada; también la historia de la arqueología continúa siendo un campo extremadamente yermo: «...the history of archaeology needs studing not only as an interesting and exciting story in itself, an account of the development of one of the newest humanistic disciplines in the historical sciences, but because withouth an historical perspective we can at the present day

BarRet-Kriegel, Les Académies de l'Histoire, Paris 1988, pág. 11

A. Momigliano, L'histoire ancienne et l'antiquaire. Problèmes d'historiographie ancienne et moderne, Paris 1983, pág. 247. 
forget, at our peril, or even repeat, past errors..." ${ }^{3}$. Así pues, tal y como señala Glyn Daniel la arqueología, entendida como estudio sistemático y descriptivo de los restos materiales de la antigüedad, necesita sustentarse sobre su propio pasado en tanto que disciplina; y por su parte, la historia de la historiografía está obligada a detenerse en consideraciones arqueológicas a la hora de abordar la evolución y características de la ciencia histórica durante el siglo XVIII, puesto que en la figura del historiador dieciochesco confluyen los perfiles del erudito, el crítico y el arqueólogo.

En este sentido, y ciñéndonos a España, eruditos, historiadores y críticos de los siglos XVII y XVIII como Ambrosio de Morales, Jerónimo Zurita, el Padre Masdeu, Enrique Flórez, Luis José Velázquez y cientos de nombres más permiten hablar de un florecimiento extraordinario de los estudios históricos en su vertiente arqueológica o de análisis de las "antigüedades", destacando sobre todo las preocupaciones numismáticas y epigráficas ${ }^{4}$.

En un orden de consideraciones paralelo nos gustaría introducir una segunda reflexión. La historiografía del XIX y del XX es heredera de un progreso anterior consistente en un movimiento de conocimiento y depuración de las fuentes que ya se detecta con claridad en La Diplomatique de Mabillon, publicada en $1681^{5}$; pero, a diferencia de la historia literaria, donde la renovación se liga fundamentalmente al talento individual, la investigación histórica entronca poderosamente con una colectividad científica, tal y como Barret-Kriegel demuestra para el caso de Francia.

Todas estas ideas pueden sugerir al investigador gran cantidad de temas y de enfoques, entre ellos los que vertebran el presente trabajo: se trataría de aproximarnos a la realidad historiográfica del siglo XVIII es-

3 Glyn Daniel, "Introduction: The necessity for an historical approach to Archaeology", Towards a History of Archaelogy. Being the papers read at the first conferences on the History of Archeology in Aarhus, 29 august - 2 september 1978, London, Thames and Hudson, 1981. De este mismo autor resulta igualmente recomendable la obra Historia de la arqueologia, de los anticuarios a Gordon Childe, Madrid, Alianza Editorial, 1974.

${ }_{4}$ Nuestra investigación bibliográfica nos ha descubierto algunas obras de interés sobre lo que podriamos denominar historia de los estudios arqueológicos españoles en el siglo XVili. Es el caso de: Antonio Beltran Martínez, "Evolución histórica de la Arqueología española", Análisis e investigaciones culturales, 24, 1985, pág. 13-24; Antonio ELIAS DE MoLins, Los estudios históricos y arqueológicos en Cataluña en el siglo xvill. Discurso de recepción en la Real Academia de Buenas Letras de Barcelona, Barcelona 1903. Otros muchos no pasan de ser meramente descriptivos o simplemente anecdóticos; vid. F. AGUILAR PINAL, Bibliografía de estudios sobre Carlos III y su época, apartado "Ciencias particulares. Arqueologia", pág. 251-252, Madrid, CSIC, 1988.

5 BarRet-Kriegel, obra citada, pág. 7. 
pañol a través de la labor de la Real Academia de la Historia, pero no partiendo de ella como objeto de estudio, sino desembocando en esta institución y en sus miembros a raiz de nuestro interés por remontarnos a los origenes de lo que más tarde serian disciplinas auxiliares de la historia o incluso ciencias independientes como la numismática, la paleografía, la epigrafía o la arqueología.

\section{LA REAL ACADEMIA DE LA HISTORIA: REFORMA Y UTOPIA}

Dentro de un movimiento académico que perseguía la renovación de la cultura en sus más variadas manifestaciones (lengua, literatura, ciencias, artes) ocupan un lugar preeminente aquellas corporaciones que tuvieron como norte renovar los estudios históricos; no hay que olvidar que uno de los elementos definidores del movimiento ilustrado es, precisamente, el despertar de una nueva conciencia histórica, que se viene a sumar a la consideración de esta disciplina como instrumento práctico y crítico indispensable para la transformación social y el beneficio de la nación ${ }^{6}$.

Así pues, las Reales Academias, entre ellas la de la Historia, fueron instituciones típicamente ilustradas en cuyo seno se expresaron inquietudes de reforma intelectual, económica y política, y en las que la monarquía seria no sólo inspiradora, sino también principal beneficiaria de su obra.

No vamos a considerar aquí cuestiones tales como la estructura y organización corporativas o las relaciones que unieron a la Academia con los poderes públicos, pues ya han sido ampliamente abordadas en trabajos anteriores ${ }^{7}$; pero sí nos detendremos en el carácter y significación

6 J. A. Maravall, "Mentalidad burguesa e idea de la Historia en el siglo Xvili", Revista de Occidente, n. ${ }^{\circ} 107,1972$, pág. 250-286.

7 Maria Teresa Rodríguez, Reformismo ilustrado y americanismo: La Real Academia de la Historia, 1735-1792, Madrid, Servicio de Publicaciones de la Universidad Complutense de Madrid, 1989.

- «La Real Academia de la Historia como modelo de unión formal entre el Estado y la cultura", Cuadernos de Historia Moderna y Contemporánea, n. ${ }^{\circ} 8,1987$, pág. 127-155.

-: “Contribución a la metodología de los estudios histórico-historiográficos: La Real Academia de la Historia en el siglo xvill", Métodos y tendencias actuales en ia investigación geográfica e histórica, Madrid, Universidad Complutense, 1988, pág. 121-129. 
de sus proyectos y realizaciones con el fin de clarificar el verdadero sentido de sus esfuerzos por localizar, recuperar y estudiar objetos antiguos.

En torno a los años 1735-36 la Junta que daría origen a la Real Academia de la Historia proyecta la realización de un Diccionario Histórico Crítico Universal de España ${ }^{8}$ en el que, dentro de un total de veintiséis materias, tiene cabida el tratamiento de temas como archivos, ruinas antiguas, pinturas, tapicerías, joyas y otras alhajas ${ }^{9}$, considerados con el objeto de proporcionar un mejor conocimiento de los asuntos básicos: genealogia, leyes, costumbres, patronato real, religiones, etc... Este proyecto, que intentaba abarcar «quanto general y particularmente se ha escrito de España sagrado y profano", recogiendo asimismo las divergencias, contradicciones y distintos puntos de vista que la investigación pudiera revelar, nos habla del nacimiento de una actitud historiográfica novedosa que en su firme objetivo de indagar y aclarar los puntos oscuros de nuestro pasado no tendría más límites que los derivados del respeto a las tradiciones y a la piedad religiosa: «En los puntos de tradición, y que piadosamente estan recividos, es menester manejarse de forma que (...) queda indemne la reputacion con los eruditos, y no haia que recelar de los Inquisidores" ${ }^{10}$.

Por una Real Cédula dada en Buen Retiro el 17 de junio de 1738 esta Junta particular de eruditos, llamada por entonces Academia Úniversal, pasaba a convertirse en una institución oficial protegida por la monarquía borbónica, cuya finalidad iba a ser, según hace público en sus estatutos, «la formación de unos completos annales, de cuio ajustado y copioso indice se forme un Diccionario, Historico, Critico, Unibersal de España, y sucesivamente quantas Historias se crean utiles para el maior adelantamiento, tanto de ciencias, como de artes, y literatos, que historiadas se hacen sin duda mas radicalmente comprehensibles" ${ }^{1 !}$.

Incluso antes de obtener la sanción real, los académicos ya habian manifestado diversidad de opiniones en cuanto a la forma de concebir el método de trabajo. Fue Juan Antonio de Rada quien en la reunión del

\& Idea del Diccionario Crítico-Histórico-Español escrita por Agustín de Montiano, 1735 , Archivo de la Real Academia de la Historia (ARAH), 11/8035.

9 Veintiséis cédulas de división de materias para el Diccionario Histórico, 1736, ARAH, $11 / 8035$.

10 Idea del Diccionario Crítico-Histórico-Español..., f. $1 \mathrm{v}$.

1 Los Estatutos se recogen íntegramente en la Real Cédula de fundación que se guarda en el despacho del actual Secretario Perpetuo de la institución. Se puede consultar una copia en la misma, que no incluye los Estatutos, en el legajo 11/8167 del ARAH. 
día 5 de mayo propuso la creación de un "Aparato" que sirviera de guía para realizar los Anales y el Diccionario o "como norte preciso de su acierto" ${ }^{12}$. Los trece apartados de que debería constar este Aparato son la muestra más clara de las inquietudes históricas que impulsaban a la corporación; uno se dedicaría a tratar la historia general y sus utilidades. ¿No está ello en consonancia con la mentalidad historicista de la época, y dado lo temprano de la fecha, no supone una importante novedad en el panorama historiográfico español? Se constata también la preocupación por el estudio y conocimiento de las fuentes primarias -medallas, inscripciones, privilegios, cronicones falsos- y el convecimiento de que se deben aclarar, de entrada, los sucesos más lejanos en el tiempo; por ello se incluyen apartados como el primer poblador, la lengua primitiva y la geografía antigua. Temas como la historia natural, la religión, las costumbres y la geografía moderna nos hablan de la variedad de aspectos considerados dentro del ámbito de lo histórico; el saber de la época aspira a la globalidad. Junto a todos estos elementos se incluyen también en el Aparato las reglas criticas y el método, considerados garantía del orden y de la veracidad de los resultados que se pensaba obtener. De todas estas partes sólo serian atendidas finalmente la historia natural, la geografia, la cronología y las medallas ${ }^{13}$.

Tras su fundación, la Real Academia de la Historia dio muestras de renovado vigor en su empeño de reformar los estudios históricos para acabar con el error, la superstición y el delirio que contaminaban la mayor parte de la herencia historiográfica anterior; pero debía salvar un obstáculo que amenazaba con sepultarla en la utopía. La realización de los Anales y el Diccionario no era viable sin antes culminar una inmensa tarea de acopio bibliográfico y de fuentes, hecho que a la larga iba a condicionar de forma clave el balance de su producción histórica. En este sentido cabría apuntar como caracteristica básica de la actividad corporativa durante el siglo XVIII una notable desproporción entre la teoría y la práctica, o dicho de otra manera el contraste entre el gran número de proyectos aprobados y unos resultados de mucha menor relevancia.

Entre 1739 y 1743, después de abandonar los trabajos sobre el Diccionario, los académicos se dedican únicamente a los temas del Aparato. El 8 de abril de 1743 la corporación abandona temporalmente esta dedicación y pasa a centrarse de nuevo en la confección de cédulas para el

12 Fastos de la Real Academia de la Historia, Madrid, Antonio Sanz, 1739, vol. I, pág. 46.

13 Vicente Castañeda Alcover, El monetario de la Real Academia de la Historia en el siglo XVIII, Madrid, Maestre, 1950. 
Diccionario, a la par que se discute la adopción del método más adecuado para su realización efectiva. Sin embargo, a pesar de los esfuerzos, las sucesivas defensas e impugnaciones de planes, entre ellos los del Conde de Torrepalma y Miguel de Medina ${ }^{14}$, acabarán desembocando en la paralización de esta iniciativa.

Otros muchos proyectos ${ }^{15}$ se irian convirtiendo, a partir de entonces, en objetivos prioritarios de los afanes académicos. Fueron planes de muy diversa índole, casi siempre ambiciosos y que presentan, además, independientemente de sus particularidades, una orientación común: responden a la imposibilidad de avanzar en la obra del Diccionario sin antes obtener un conocimiento fidedigno de las fuentes y en relación con ellos se irán promoviendo actividades complementarias que, cada vez más alejadas de los ideales originarios, irán conquistando, lentamente, métodos y fines privativos; nos referimos a la localización de documentos y antigüedades, a la acumulación de noticias y a la organización de viajes de reconocimiento de archivos. Todo ello no empaña, a pesar de todo, el mérito de otros logros académicos: la defensa de una historia-verdad basada en la crítica objetiva de fuentes o la concepción de obras tan ambiciosas como el Diccionario Histórico, la Cronología, el Diccionario Geográfico o la Historia General de las Indias justifican sobradamente el protagonismo de la Real Academia de la Historia en el panorama historiográfico de nuestra llustración, aún cuando debamos reconocer que nuestro siglo XVIII español apenas superó la fase de construcción de cimientos del edificio histórico.

14 La documentación relativa al enfrentamiento entre Medina y Torrepalma es la siguiente: Principios del proyecto general del Diccionario histórico por el Conde de Torrepalma a quien se encargo desde el tiempo de la Junta y Representación de $D$. Miguel de Medina a la Academia manifestando los inconvenientes que le parecía tener el método de trabajar el Diccionario histórico y proponiendo la idea de otro nuevo método, Madrid, diciembre de 1744, ARAH, 11/8035. Proyecto de Diccionario Histórico que formó $D$. Miguel de Medina y aprobó la Academia en 1745 y opiniones de algunos académicos; Voto de $D$. Alonso Verdugo conde de Torrepalma oponiéndose al proyecto del Diccionario Histórico que formó el Sr. Medina, 21 de noviembre de 1745, así como la Respuesta de Medina al voto de Torrepalma, diciembre de 1745, ibidem.

${ }_{15}$ María Teresa Nava Rodriguez, "Logros y frustraciones de la historiografía ilustrada a través de los proyectos de la Real Academia de la Historia", Coloquio Internacional Carlos III y su siglo organizado por el Departamento de Historia Moderna de la Universidad Complutense de Madrid y celebrado en noviembre de 1988. (En prensa). 


\section{LAS PREOCUPACIONES “ARQUEOLÓGICAS»}

Veamos, por tanto, cuáles son las manifestaciones más claras de este impulso orientado a rescatar monumentos, entendidos éstos como vestigios escritos o materiales del pasado.

La Academia, además de recibir y examinar trabajos enviados por particulares dando cuenta de documentos y antigüedades hallados en bibliotecas y yacimientos, también promovió directamente este tipo de investigaciones comisionando a algunos de sus miembros; así, se organizaron y realizaron varios viajes con el fin de cotejar documentación o descubrir y estudiar restos materiales. En el conjunto de estas iniciativas destacan las siguientes: en primer lugar, la aportación de Lorenzo Diéguez y Pedro Rodríguez Campomanes, comisionados en 1751 para cotejar datos cronológicos sobre los reyes godos y sobre los primeros monarcas de Asturias y León en la Biblioteca del Real Monasterio de El Escorial. En 1754 y 1755 repitieron la experiencia, esta vez temporalmente acompañados por Ignacio de Hermosilla y Luis de Herrera ${ }^{16}$.

De igual, o quizá mayor interés, son las investigaciones llevadas a cabo por el académico Luis José Velázquez ${ }^{17}$. El 1 de diciembre de 1752 Velázquez inicia un viaje para «inquirir y recoger las antigüedades de

16 Papeles sobre los viajes de Lorenzo Dieguez y Pedro Rodriguez Campomanes (año 1751) a la Biblioteca del Escorial, ARAH, 9/5937. Relación del segundo y tercer viaje al Escorial en 1754 y 1755 hechos por Pedro Rodriguez Campomanes y Lorenzo Dieguez, ARAH, 9/5725-26. Papeles sobre reconocimiento de Archivos, ARAH, 9/5921.

17 Luis José Velázquez de Velasco, marqués de Valdeflores (1722-1772) es una de esas figuras de talla reconocida a las que la historiografía todavía no ha hecho justicia. Sempere y Guarinos en su Ensayo de una Biblioteca Española de los mejores Escritores del Reynado de Carlos III (Tomos V-VI, 1789, pág. 9-28) dedica un buen número de páginas a comentar sus principales obras, dejando perfectamente dibujado el perfil de una destacada producción, a la vez profunda y diversa. Ingresó como supernumerario en la Real Academia de la Historia el 5 de abril de 1751, pasando a ocupar plaza de numerario sólo un año más tarde; también en el ámbito de la vida corporativa la participación de Velázquez es significativa. Por todo ello tenemos intención de investigar su vida y su obra empleando de entrada los importantísimos fondos que se conservan en el Archivo de la Academia (Colección Velázquez, signaturas 9/4105 a 9/4159) y en la Biblioteca Nacional (Colección del Duque de Osuna): copias y apuntes sobre documentos, comentarios acerca de monedas y medallas, construcciones cronológicas, disertaciones, monografías y un largo y sugerente etcétera. 
todo el Reyno" ${ }^{18}$; partía con la orden expresa de escribir puntualmente al director de la Academia dando cuenta de sus trabajos, noticias que el propio director debería ir transmitiendo al rey. Velázquez visitó Mérida, Salamanca, Córdoba, Jaén, Granada, Sevilla y Málaga, remitiendo a la corporación, a principios de 1755 , unas memorias en las que comentaba las características de las antigüedades que había localizado. A principios de 1753 y en cumplimiento de una orden real, la Academia tuvo asimismo que elaborar un informe sobre la espada hallada en el muro de un convento de Peñafiel ${ }^{19}$.

La necesidad de aclarar y probar la veracidad de muchos hechos, así como el interés creciente por las antigüedades ${ }^{20}$, hizo que la Academia financiara nuevos viajes en la década de los sesenta: las estancias de Lorenzo Diéguez y Alonso Cano en la Biblioteca Real de Madrid y en las de El Escorial, Colegio Mayor Alcalá y Catedral de Toledo en 1762, el reconocimiento que hizo Ignacio de Hermosilla del terreno y antigüedades de Talavera la Vieja ese mismo año y la investigación llevada a cabo por Alsinet sobre las antigüedades de Cabeza del Griego ${ }^{21}$.

La importancia de la labor de acopio de fuentes alcanza su punto álgido en 1755 con la propuesta de Pedro Rodríguez Campomanes en torno a la formación de un Índice Diplomático de España. Campomanes ya había sugerido, en 1753, emprender una obra de recopilación de fuentes citadas en historias generales o particulares de España, incluidas las

18 Las Memorias de la Real Academia de la Historia recogen la noticia de este viaje (Madrid, Sancha, 1976, vol. I, pág. XXXVII). Para conocer más detalles se puede consultar la Noticia del Viage de España hecho por orden del Rey y de la nueva historia general de la nacion desde el tiempo mas remoto hasta el año 1516 sacada únicamente de los escritores y monumentos originales, y contemporáneos con la coleccion universal de estos mismos escritores, $y$ Monumentos recogidos en este viage por D. Luis Josef Velazquez, Madrid, Gabriel Ramirez, 1765, (ejemplar en la Biblioteca del Centro de Estudios del Siglo XvIII de Oviedo). Vid. además el legajo misceláneo número 9/7018 del ARAH.

19 Sobre la espada encontrada en el Convento de San Juan y San Pablo de Religiosos Dominicos de la villa de Peñafiel, ARAH, 9/5930.

20 Florencio JANER, «Aprecio en que se tenian los objetos científicos y arqueológicos en el reinado de Carlos III y Carlos IV", Revista de España, XVII-XVIII, 1870, pág. 200227.

${ }^{21}$ En torno a las excavaciones realizadas en Cabeza del Griego hemos reunido una información muy detallada que esperamos elaborar y publicar en breve. La mayor parte proviene de la documentación conservada en el Archivo de la Academia, legajos 9/5939 y 9/4130. Almagro BASCH hace referencia a ellas en su obra Segóbriga (ciudad celtibérica y romana). Guía de las excavaciones y Museo, Madrid, Museo Monográfico de Segóbriga, 1978, al igual que siglo y medio antes lo hiciera CEÁ-BERMúdez en el Sumario de las Antigüedades Romanas que hay en España, en especial las pertenecientes a las Bellas Artes, Madrid, Miguel de Burgos, 1832. 
fuentes litológicas, pero, debido a la lentitud con que se desarrollaban los trabajos, el cuerpo acabó decidiendo en mayo de 1755 limitar la colección a las «inscripciones, epitafios, y otros letreros de cualquier tiempo, caracter, y lengua que se hayasen" ${ }^{22}$.

Por entonces la Academia era ya plenamente consciente de la imposibilidad de escribir con propiedad acerca de cualquier asunto histórico sin contar con una colección adecuada de noticias generales sobre historiadores coetáneos y sobre fuentes; también pensaba que con ello se facilitarían los trabajos sobre cronología, cuyo reto principal era confrontar datos en libros, memoriales, genealogías y monumentos de los que no se tenía un conocimiento exhaustivo y ordenado.

Por fin, el 18 de agosto de 1755 se aprobó la formación de un índice Universal Diplomático de España ${ }^{23}$, pasándose luego a confeccionar las reglas que debian seguirse tanto en este índice como para el litológico. El resultado sería satisfactorio, y en torno a 1760 se habia logrado reunir más de cincuenta y seis mil cédulas.

La orientación erudita, de impronta bibliográfica, archivística o arqueológica, se fue asentando cada vez más sólidamente en el marco de las actividades corporativas hasta el momento de aprobación de unos nuevos estatutos en $1792^{24}$. Otros proyectos secundarios así lo demuestran. A partir de 1764 el número de planes propuestos se incrementa vertiginosamente, y entre ellos algunos que pueden servir de ejemplo: la acuñación de una colección de medallas de nuestros reyes (1765), la formación de una historia metálica de Carlos III (1766), la elaboración de una Paleografia y una Bibliografía españolas en las que trabajarian académicos como Abad y Lasierra y Francisco Javier Palomares - Casiri la completará con una Paleografia arábiga y de inscripciones cúficas en 1787-y, por último, en abril de 1790, el diseño de las inscripciones que decorarian la Torre de Hércules de La Coruña, previo encargo encomendado a la Academia por el Ministerio de Marina.

22 Memorias de la Real Academia de la Historia, vol. I, pág. XLII.

${ }^{23}$ Instrucción que forma la Academia de la Historia sobre el uniforme método de sus individuos en las cedulas para el Indice Universal Diplomático de España, Archivo Histórico Nacional, Estado, legajo 4819. Véase también la «Proposición para un Indice chronologico de todos los privilegios, Bulas, ò Diplomas que hace a la Real Academia de la Historia Don Pedro Rodriguez Campomanes Academico Numerario y uno de sus tres Revisores", Libro primero de entrada y revisión de cédulas tocantes al Indice diplomático de España, 1755 , ARAH, 11/8360.

${ }^{24}$ Nuevos Estatutos de la Real Academia de la Historia. Aprobados por S. M. por Real Resolución de 15 de Noviembre de 1792, a consulta de la Academia de 4 de octubre del mismo año. (Ejemplares en la Biblioteca Nacional y en el ARAH). 
En un nivel similar de importancia al de los datos hasta aquí recogidos sobre planes generales, se encuentra la constatación del enorme número de memorias, disertaciones, discursos e informes que a título individual, pero con justificación corporativa, realizaron algunos de sus individuos. El análisis temático de estas obras ${ }^{25}$ arroja un balance meridiano. De un total de ciento veintiseis trabajos considerados, dieciséis abordan temas relacionados con el hallazgo de la situación geográfica, aún no identificada, de lugares mencionados por los historiadores antiguos y dieciocho presentan un enfoque fundamentalmente arqueológico, numismático o litológico, hasta tal punto relacionado con la perspectiva anterior, que en ocasiones ambos aspectos se superponen o complementan en una misma obra ${ }^{26}$. También podrían relacionarse con este área temática algunos trabajos que, si bien entran con mayor propiedad en el campo de la historia política, tienen como objetivo básico averiguar cuál fue la patria de algún personaje importante, o el lugar de localización de batallas y otros acontecimientos ${ }^{27}$. La cercanía, o incluso dependencia conceptual entre la historia propiamente dicha y materias como la geografia "antigua», la arqueología, la numismática y la epigrafía quedarian aquí perfectamente ejemplificadas.

"Vacios estamos pues y fatigados al cabo de media centuria de pasearnos por tantos proyectos literarios, cogiendolos por antojo y sin sistema y descuidandolos sin examen ni escrupulo. Pero la Academia (...) es un cuerpo muy respetable, dotado y muy protegido por el Govierno con el que contraxo obligaciones que le estrechan (...) somos el obgeto de un publico que no perdona a nadie y que no se engaña que cuenta los años de nuestro silencio por los de nuestra existencia» ${ }^{28}$.

En torno a 1792 se vió llegado el momento de meditar seriamente sobre las obras que debían ser continuadas o emprendidas y de qué

25 María Teresa NAVA RODRIGUEZ, «Producción historiográfica y academicismo durante el reinado de Carlos III: Discursos de entrada, disertaciones y otros escritos", IV Encuentro de la llustración al Romanticismo, abril 1988 (en prensa).

${ }^{26}$ Es el caso de la obra de JOSE CORNIDE titulada Disertación sobre cual hubiese sido el Antiguo asiento de la ciudad Limica o Lemica señalada por patria de ldacio en el prologo de su cronicón, 2 de mayo de 1755, ARAH, 9/5996. En ella Cornide intenta descubrir la verdad sobre este punto oscuro de la geografía antigua sirviéndose de algunas inscripciones que él mismo habia encontrado y analizado.

${ }_{27}$ Juan Martinez Salafranca, Sobre el lugar que los historiadores griegos y latinos dicen que Alejandro venció a Darío, último rey de los persas, 9 de marzo de 1735, ARAH, 9/5991.

${ }_{28}$ Memoria de lo emprendido y trabajado por la Real Academia de la Historia desde 1739 hasta 1792, ARAH, 9/4181, ff. 20 r.-20 v. 
forma llevarlas a cabo. Para lograr la regeneración de la Academia era necesario mejorar el método y el orden de los trabajos yendo a lo fundamental sin intentar abarcar todo, y junto a ello, elaborar unos nuevos estatutos más claros y extensos que permitieran desterrar las acciones arbitrarias del funcionamiento institucional.

La Junta extraordinaria encargada de llevar a cabo la reforma acabó seleccionando seis únicos proyectos: " $1^{\circ}$ Las excerptas de los Autores originales y primitivos de cosas pertenecientes á España, puestos por orden cronológico, (...) $2^{\circ}$ La colección de los Primeros Autores de nuestra historia: $3^{\circ}$ La prosecución de la colección de cédulas diplomaticas y litologicas: $4^{\circ}$ Discursos sobre puntos dudosos de nuestra historia: $5^{\circ}$ Una diplomatica española: $6^{\circ}$ Una biblioteca historica de España» ${ }^{29}$. Esta elección no hacía más que sancionar oficialmente una situación de hecho, orientando a la Academia de la Historia hacia tareas para las que sí habia demostrado aptitud y eficacia. Su labor, además de tener un peso teórico y metodológico, permite a su vez una lectura práctica y numérica: durante el siglo XVIII logró reunir " 13.500 documentos originales para la historia de España, 7.000 diplomas, más de 4.000 inscripciones, 2.000 medallas y 62 monumentos de pintura, escultura y arquitectura" ${ }^{30}$, lo cual permite atribuirle un papel de primer orden entre todos aquellos, instituciones o particulares, que asumieron el ideal ilustrado de la construcción de una Historia científica.

29 Memorias de la Real Academia de la Historia..., pág. CXIV.

30 AlboRG, Historia de la literatura española, Madrid, Gredos, 1978, vol. III, pág. 34. La Real Academia de la Historia posee en la actualidad una importante colección de antigüeciades que se comenzaron a reunir en el siglo XVIII; vid. Juar Catalina GARCIA LOPEZ, "Inventario de antigüedades y objetos de arte que posee la Real Academia de la Historia", Boletín de la Real Academia de la Historia, 1903, tomo 42, pág. 311-316, 321-368 y 484-505; 1903, tomo 43, pág. 257-322. Por último, dar noticia al lector de la existencia de una Colección litológica formada por la Academia de la Historia, por el orden alfabético de los pueblos en que se encuentran las inscripciones, ARAH, 9/6500-6513. 